\title{
Entomopathogenic organisms: conceptual advances and real-world applications for mosquito biological control
}

This article was published in the following Dove Press journal:

Open Access Insect Physiology

20 April 2016

Number of times this article has been viewed

\author{
Richard I Samuels' \\ Adriano R Paula' \\ Aline T Carolino' \\ Simone A Gomes' \\ Catia OP Morais' \\ Mariana BC Cypriano' \\ Leila El Silva' \\ Anderson Ribeiro' \\ Jonathan WAB Santos' \\ Carlos P Silva ${ }^{2}$ \\ 'Department of Entomology and Plant \\ Pathology, State University of Norte \\ Fluminense, Campos dos Goytacazes, \\ Rio de Janeiro, ${ }^{2}$ Department of \\ Biochemistry, Federal University of \\ Santa Catarina, Florianópolis, Brazil
}

Correspondence: Richard lan Samuels Department of Entomology, State University of North Fluminense, Avenida Alberto Lamego 2000, Campos dos Goytacazes, Rio de Janeiro, CEP 280।3-602 Brazil

Tel +55 2I 22999620662

Email richardiansamuels@gmail.com

\begin{abstract}
Recently, advances have been made in the use of entomopathogenic fungi for the control of not only crop pests but also disease vectoring insects. New approaches, for example, combining control strategies such as the application of conventional insecticides and biological control agents together, are highly promising, especially as many insecticides are compatible with entomopathogenic fungi. In this review, we discuss some of the new approaches being developed for vector control. Governmental authorities must implement rational integrated vector management programs for reducing deaths and suffering caused by insect-vectored diseases. Although there are now many alternatives to conventional chemical control methods, the use of pesticides is still the mainstay of mosquito control measures. However, mosquitoes are rapidly developing resistance to the chemical insecticides in use at the moment. The deployment of entomopathogenic fungi for the control of all stages of the mosquito life cycle is an alternative strategy currently being investigated by many different research groups. It has been shown that entomopathogenic fungi can efficiently kill mosquito larvae and adults in laboratory and field conditions. However, an important aspect of this new approach is how to apply these biological control agents economically and efficiently. By using combinations of control agents and novel application systems, it may be possible to significantly reduce mosquito populations to levels at which epidemics of malaria, dengue fever, zika virus, and chikungunya do not occur.
\end{abstract}

Keywords: entomopathogenic fungi, insect, disease vector, insecticide, biological control, dengue, zika

\section{Introduction}

Entomopathogenic fungi are common and widely dispersed among insect species. ${ }^{1}$ Extensive research has been conducted on a range of Deuteromycete fungal genera (Beauveria, Culicinomyces, Tolypocladium, and Metarhizium) for the biocontrol of many agricultural pests, ${ }^{2}$ and although these fungi have a limited impact on natural populations of mosquitoes in the field, Beauveria bassiana (Bals. -Criv.) Vuill. 1912 and Metarhizium anisopliae (Metschn.) Sorokin 1883 have potential for use in vector control. ${ }^{1} M$. anisopliae and B. bassiana are entomopathogenic fungi whose conidia can infect insects by penetrating the cuticle, without needing to be ingested, causing death in a matter of days. ${ }^{3}$ These species are found distributed on a worldwide basis and do not harm birds, fish, or mammals. ${ }^{4}$

The infective process of entomopathogenic fungi is based on three sequential phases (Figure 1). The first phase is characterized by adhesion and germination of conidia on the insect's cuticle, following which the germ tube penetrates the integument. Finally,

submit your manuscript | www.dovepress.com 
after invading hemocoel, the fungus develops in the hemolymph and other internal tissues (colonization), leading to death of the host. ${ }^{5}$

Their specialized mechanism of infection through active penetration of the host integument gives these fungi an advantage over other biological control agents such as toxinproducing bacteria, protozoa, and viruses, as direct contact of conidia with the cuticle of the host is necessary to initiate the process of infection. Therefore, fungi can infect all stages of the insect's developmental cycle from egg to adult, whereas other types of pathogenic microorganisms need to be ingested to initiate an infection. ${ }^{5}$ Due to the development of pesticide resistance in insects, it is, therefore, extremely important to research alternatives for vector control.

One of the major obstacles to the use of filamentous fungi for biological control of insect pests is the time interval between the application and the death of the host, when compared to that of applications of chemical pesticides. One of the common goals in the study of these microorganisms is to speed up death of the host and, thus, improve the biocontrol efficiency. Accordingly, efforts have been made to improve production and stability of these fungal inocula and application technologies. ${ }^{6}$ Here we describe new approaches for vector control, combining entomopathogenic fungi with insecticides or plant bioactive compounds applied to black cloths for adult mosquito control and encapsulating fungi for application against mosquito larvae. Encapsulation technology can be used to increase the persistence of fungi and it is also possible to incorporate other insecticidal compounds/ adjuvants within the microcapsules.

\section{Problems associated with the control of Aedes aegypti}

Dengue fever is one of the most serious viral diseases vectored principally by the mosquito Aedes aegypti (Culicidae, Diptera) Linnaeus, 1762, affecting 50-100 million people annually. ${ }^{7}$ Around 500,000 people with severe dengue require hospital treatment annually, many of whom are children. Sadly, this year's epidemic in Brazil resulted in a high death rate among the older population, 169 deaths so far in the state of São Paulo, with nine out of ten victims over the age of 60 .

A new vaccine should be available this year, but will provide only around $60 \%$ protection, which means that control of the insect vector continues to be a high priority. The main strategies for the control of $A$. aegypti are elimination of breeding sites and chemical insecticide application. However, these strategies have not prevented regular epidemics of dengue fever and new approaches are, therefore, urgently needed. One of the main problems associated with chemical control is the rapid development of insecticide resistance, which is particularly serious in Brazil where A. aegypti larvae

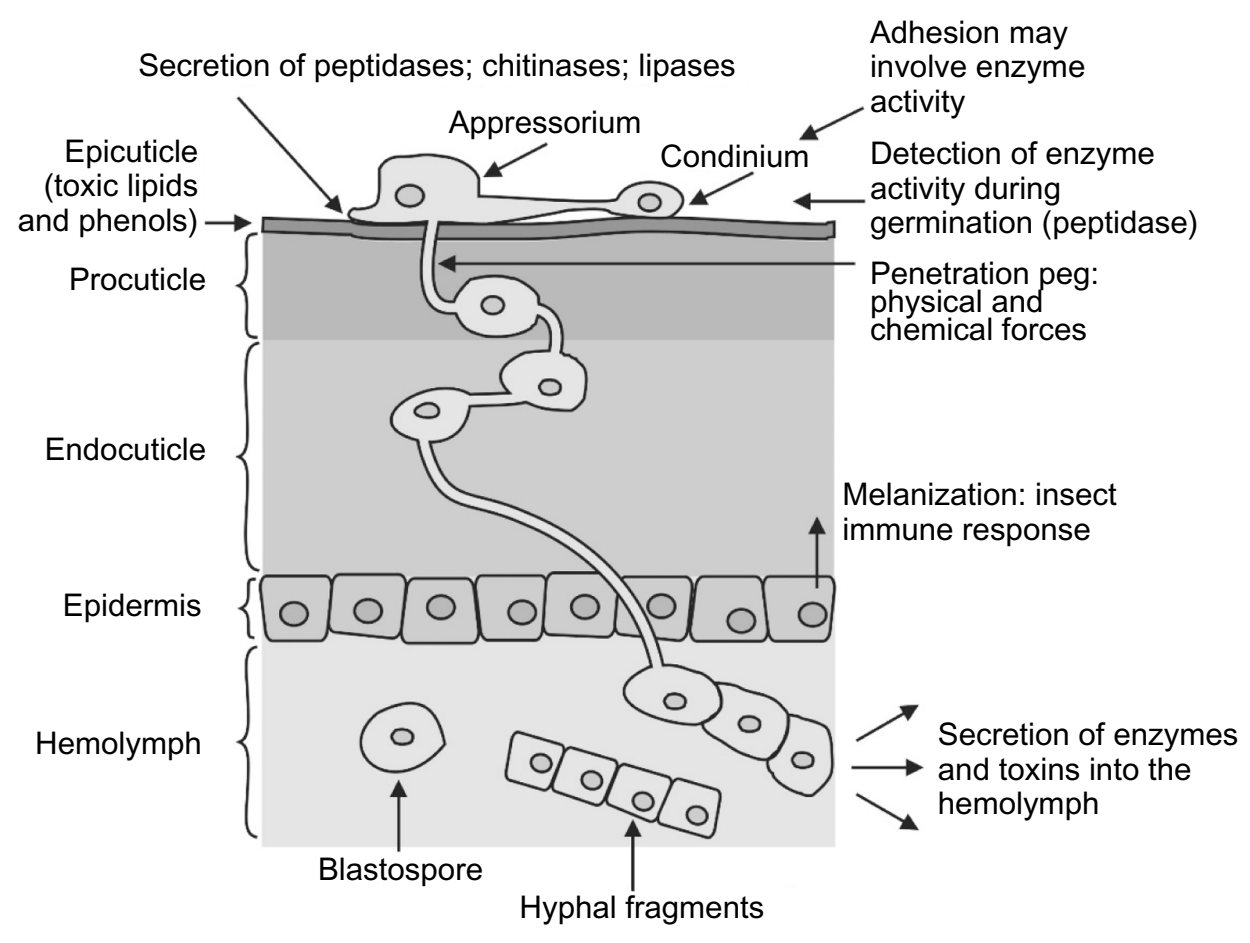

Figure I Summary of the events involved in the infection cycle of an entomopathogenic fungus attacking an insect host. 
have evolved resistance to the widely used organophosphate temephos ${ }^{8}$ and adult mosquitoes have also evolved resistance to pyrethroids cypermethrin and deltamethrin. ${ }^{9}$

\section{Combined use of entomopathogenic fungi and phytochemicals for larval control}

The insecticidal activity of phytochemicals against mosquito larvae has been well documented, ${ }^{10}$ although no commercial products based on phytochemicals are currently being used in mosquito control programs. One of the most extensively used "natural" plant-derived insecticides is neem, extracted from the plant Azadirachta indica. ${ }^{11}$

Commercially available neem-based insecticides are used to control many crop pests, and this is considered as a "green" approach to pest control, permitted in organic production systems. Among the plant bioactive compounds, neem oil is one of the few to have been commercially successful in agricultural regimens. Neem tree extracts have also been tested for toxicity against mosquito larvae and have been shown to kill Aedes, Culex, and Anopheles. ${ }^{12-14}$

Continuing our search for useful adjuvants to increase virulence or persistence of entomopathogenic fungi, we demonstrated that neem oil can significantly increase the effectiveness of M. anisopliae when applied to water containing Aedes larvae. ${ }^{15}$ This type of approach is important when aiming for rapid mortality of larvae especially as fungal conidia have a limited half-life under field conditions.

The current approach using a combination of phytochemicals and entomopathogenic fungi has multiple benefits. Apart from increasing mortality rates, neem could also increase fungal persistence and reduce the chances of resistance development which has been shown to occur when using mixtures of control agents. ${ }^{16}$

\section{Microencapsulation of entomopathogenic fungi}

Microencapsulation is a technology that uses thin polymer layers applied in solid, liquid droplets, or gaseous material, which form particles called microcapsules that release their contents under specific conditions and at different rates. ${ }^{17}$

The capsule size is classified into macro $(>5,000 \mu \mathrm{M})$, micro $(0.2-5,000 \mathrm{mM})$, and nanoparticles $(<0.2 \mu \mathrm{m})$. In terms of capsule structure, they are divided into two categories: capsules in which the core is distinctly concentrated in the central region surrounded by a wall of continuous film material and capsules in which the core is dispersed in a matrix. The first group is identified as reservoir-type system, characterizing the "true microcapsules", while the second group is classified as a matrix system, resulting in so-called "microspheres". ${ }^{18}$

When comparing the two groups, the main difference is related to the fact that in the microspheres, part of the encapsulated material remains exposed on the surface, which does not occur with the true capsules. ${ }^{19}$ The main aim of the encapsulation process is to allow the release of the core material slowly over time or during a given event. ${ }^{20}$

Among the materials used in formation of the encapsulating matrix are agar, agarose, carrageenan, collagen, alginate, chitosan, and cellulose. ${ }^{21}$ The selection of the appropriate encapsulate product is essential; it must have the ability to seal and maintain the active material in the capsule structure, completely releasing the solvent or other materials used during the encapsulation process and providing maximum protection to the active material against adverse conditions such as ultraviolet light, $\mathrm{pH}$, oxygen, and reactive species. It must also be soluble in commonly used solvents, retaining the desired properties following release of the active material.

Alginates are commonly used as encapsulation materials, which, primarily due to their gelling properties, are responsible for an important effect on the stability of the capsules. ${ }^{22}$ Chemically, alginate is composed of linear chains of $\alpha$-Lglucuronic acid and $\beta$-D-mannuronic acid, which in the presence of ions such as $\mathrm{Ca}^{++}$form hydrogels, films, beads, and microparticles with a high encapsulation capacity. ${ }^{23}$ Alginate has been widely used as an encapsulate of microorganisms, mainly due to low toxicity, biodegradability, economy, and ease of handling. ${ }^{24,25}$

Encapsulation of microorganisms protects the biocontrol agents and increases their shelf life. Furthermore, with the use of microencapsulation technology and modifications of the polymeric matrix, microspheres can be developed to achieve the desired release profile. ${ }^{25}$

Pereira and Roberts ${ }^{26}$ investigated the production of conidia of the fungi $M$. anisopliae and B. bassiana using an alginate formulation, and confirmed that this prevented degradation of the encapsulated fungi. In another study, cross-linked alginate microcapsules coated with calcium and cashew gum were prepared and injected with plant essential oil (extracted from Croton zehntneri Pax et Hoffm.) with larvicidal activity, in order to preserve the active ingredient, prolong the release time, and increase toxicity. It was observed that the polymer matrix provided effective protection, with the material remaining active for up to 70 days. ${ }^{27}$ Accordingly, microencapsulation could help in the development of biopesticides, incorporating entomopathogenic fungi. The lack 
of an adequate formulation has impeded the large-scale use of entomopathogenic fungi against mosquito larvae.

Development of new mosquito control methods through the use of microorganisms should be based on principles that make field application efficient and practical, with economically viable products and long shelf life. In this regard, further work should be carried out in order to understand the factors directly related to virulence, persistence, and viability of entomopathogenic fungi, when they are subjected to microencapsulation technology.

\section{Adult mosquito control using fungus-impregnated surfaces}

Conventional adult disease vector control relies on three methods of application: 1) indoor residual spraying, 2) space spraying, and 3) long-lasting insecticidal (bed) nets (LLINs). Indoor residual spraying and LLINs have been highly successful in Africa for the control of mosquito species that vector malaria. ${ }^{28}$ Spraying of persistent insecticides onto surfaces which the mosquitoes land has saved thousands of lives. Insecticides can be sprayed onto the walls of houses or applied to bed nets. Only pyrethroid insecticides are approved for use on LLINs.

Conversely, the development of insecticide-resistant mosquito populations is rapidly becoming one of the most serious problems facing the authorities. ${ }^{29}$ Entomopathogenic fungi can be used against mosquito populations that have already developed resistance to conventional chemical insecticides. ${ }^{30,31}$ Even so, biological control strategies will not replace chemical control. However, it should also be remembered that strategies for chemical control of dengue vectors are very different from those used against malaria vectors due to distinct behavioral differences between the species. Dengue mosquitoes do not blood feed at night, but they do so in the morning and at nightfall. Currently, in Brazil, adult $A$. aegypti management is carried out using chemical insecticides that are normally applied using ultra-low-volume space spraying equipment.

We have proposed that entomopathogenic fungi could be viable alternatives to chemical control methods, but can also be used in combination with chemical insecticides. This approach could prolong the useful lifetime of insecticides. The combined use of fungi and insecticides can increase efficiency by synergism, reducing the time to kill. ${ }^{32,33}$

One of the most important aspects of using fungi for vector control is the development of efficient delivery methods. An alternative to spraying is the application of fungal propagules to surfaces onto which mosquitoes are known to land. One method which has been tested in Africa is the use of fungus-impregnated cloths hung from the ceilings of houses. ${ }^{34}$ Recently, fungi have also been applied to the inside of clay water storage pots. ${ }^{35}$ As mosquitoes pass through the eaves of houses when attempting to find their human hosts, fungus applied to eave curtains could also be effective for the infection of adults. ${ }^{36}$

Our group has concentrated on the use of small black cotton cloths $(\sim 8 \times 6 \mathrm{~cm})$ impregnated with conidia as a potential delivery system for use in intra- and extra-domicile situations. This is a simple delivery system for biological control of adult mosquitoes, directed at their known behavioral preferences for resting/landing on dark surfaces. ${ }^{37}$ We believe this approach can be cost-efficient and highly effective. We have shown this method to be effective under field simulation conditions, significantly reducing the survival times of female $A$. aegypti. ${ }^{38}$

We initially tested fungus-impregnated black cotton cloths hung in large cages into which female A. aegypti had been released. The survival curves of control and fungus-treated insects were significantly different. ${ }^{39,40}$ The longer the cloths are left in the cages with the mosquitoes, the shorter the life span of the insects. This shows that there is a time-dosedependent inoculum pickup. ${ }^{40}$

It is important to note that the fungus-impregnated cloths did not alter the landing behavior of the mosquitoes. This has been confirmed by studies which have shown that mosquitoes are not repelled by fungus-treated surfaces. ${ }^{41}$ However, we did observe low landing/resting frequencies during scotophase. Landing on dark surfaces could be a defense mechanism of the adult mosquitoes against predators, as they are very difficult to be seen when resting on these surfaces during daylight hours.

A 39\%-57\% reduction in malaria mosquito survival was seen when using fungus-treated baffles attached to the eaves of houses or fungus-treated strips of cloth placed around an occupied bed net. ${ }^{42}$ Using a different approach, which did not involve exposing humans to potential allergies from close contact with fungal conidia, bait stations were set up in the field around villages, containing attractive baits and fungi. The results of this study showed that $95 \%$ of female Anopheles arabiensis that entered the bait stations became infected with fungi within 14 days of exposure. ${ }^{43}$ The idea of combining entomopathogenic fungi with attractive baits is an interesting approach to maximize the chances of the vector coming into contact with the fungus. This approach has been successfully commercialized for cockroach control. $^{44}$ 
Tests carried out in simulated field conditions in Australia showed that exposure of $A$. aegypti to $B$. bassiana caused a reduction in survival (59\%-95\%), blood-feeding behavior, and fecundity. ${ }^{45}$

The next section will further describe the combined use of fungi and conventional insecticides.

\section{Combined use of entomopathogenic fungi and conventional insecticides for adult control}

This is an interesting approach, as the combined use of insecticides with fungi can significantly reduce vector survival times, even when using "sublethal" concentrations of insecticides. Many insecticides are compatible with entomopathogenic fungi and our research group has been testing the combined effects of imidacloprid (IMI) with $M$. anisopliae. Germination and growth assays of the fungus in the presence of the insecticide demonstrated that there were no adverse effects of the insecticide on these two parameters. ${ }^{46}$ The use of so-called sublethal concentrations of insecticide (defined as a concentration that causes no statistically significant alteration in the survival curves, when compared to the controls) reduced mean survival times from 3 to 2 days. ${ }^{33}$ It should be remembered that our aim is not necessarily to cause rapid mortality of the mosquito, but maximize the chances of the fungus to initiate an infection. Furthermore, studies of fungal infections of malaria mosquitoes have shown that relatively small changes in survival rates were sufficient to significantly reduce vectorial capacity. ${ }^{6}$

Dengue virus infected insects could be more susceptible to fungal infection, as A. aegypti became immunocompromised due to downregulated expression levels of numerous immune signaling molecules and antimicrobial peptides, ${ }^{47} \mathrm{a}$ fact that could benefit the use of entomopathogenic fungi as biological control agents.

Insecticides have the capacity to increase stress and alter insect behavior, which may lead to improved performance of entomopathogens. ${ }^{48,49}$ IMI is not currently being used as a chemical control agent of adult or mosquito larvae, but has been tested against three mosquito species, including $A$. aegypti. ${ }^{50}$ Another study demonstrated that neonicotinoids such as IMI were toxic to larval and adult $A$. aegypti, although IMI was only considered to be an interesting candidate for larval control. ${ }^{51}$

Our results show the positive effect of sublethal concentrations of IMI on fungal virulence. Significant differences were observed in mean survival percentages following shortterm contact with fungus-impregnated filter paper + IMI, when compared to the results for mosquitoes exposed to the fungus alone. Although we consider M. anisopliae to be highly virulent against $A$. aegypti, it is important to take into consideration the necessity to optimize the chances of initiating a fungal infection, for which we are now testing synthetic attractive baits to work in harmony with the fungus. One should remember that any small reduction in mosquito survival can have significant consequences of the transmission of arboviruses.

\section{Conclusion}

Here we demonstrate the potential of some of the new approaches to mosquito control based on the use of entomopathogenic fungi. When considering larval control, it is important to remember that dengue mosquitoes normally oviposit in water with low levels of organic material, different from Culex, which have a preference for stagnant water. This can be considered an advantage for the control of Aedes, as oviposition sites can be readily identified and treated with insecticides, whereas Culex can cover huge areas, for example, swampland, as breeding sites. In that case, it would be difficult to target these areas with entomopathogenic fungi and the quantities of inoculum needed would be economically prohibitive. The use of technologies such as microencapsulation of fungal conidia is an interesting approach for the control of the aquatic stage of the vectors' life cycle. However, much work is still needed before large-scale field tests can be carried out. Combining low concentrations of insecticides or phytochemicals with conidia in microcapsules could not only increase efficiency of the fungus, but could also increase their shelf life.

Targeting adult dengue mosquitoes with entomopathogens would appear to be complicated, as these entomopathogens cannot replace conventional insecticides applied to bed nets. Dengue mosquitoes are daytime feeders; therefore, treating bed nets would be ineffective. However, spraying walls with fungi could be an interesting approach, although less-random strategies could be more efficient. The use of black cloths treated with fungal conidia is currently being tested in the field by our group in a first-of-its-kind project to reduce dengue mosquito populations. Again, the fungi can be formulated with conventional insecticides to reduce time to kill and we are also testing synthetic attractants attached to the cloths in order to increase the time the mosquitoes spend resting on these fungus-treated surfaces. The results are very promising and with new formulation technology, we hope that the black cloths can be left in the field for up to 1 month. Efficient control of $A$. aegypti is not simple and the best options are combinations of control strategies in "integrated vector management". 


\section{Disclosure}

The authors report no conflicts of interest in this work.

\section{References}

1. Scholte EJ, Knols BGJ, Samson R, Takken W. Entomopathogenic fungi for mosquito control: a review. $J$ Insect Sci. 2004;4:19.

2. Shah PA, Pell JK. Entomopathogenic fungi as biological control agents. Appl Microbiol Biotechnol. 2003;61:413-423.

3. Roy HE, Steinkraus DC, Eilenberg J, Hajek AE, Pell JK. Bizarre interactions and endgames: Entomopathogenic fungi and their arthropod hosts. Annu Rev Entomol. 2006;51:331-357.

4. Zimmermann G. The entomopathogenic fungus Metarhizium anisopliae and its potential as a biocontrol agent. Pest Sci. 1993;37:375-379.

5. Charnley AK. Fungal pathogens of insects: cuticle degrading enzymes and toxins. Adv Bot Res. 2003;40:241-321.

6. Thomas MB, Read AF. Can fungal biopesticides control malaria? Nat Rev Microbiol. 2007;5:377-383.

7. World Health Organization: Dengue and severe dengue. Fact Sheet No. 117 [updated May 2015]. Available from: http://www.who.int/ mediacentre/factsheets/fs117/en/. Accessed June 15, 2015.

8. Montella IR, Martins AJ, Viana-Medeiros PF, Lima JB, Braga IA, Valle D. Insecticide resistance mechanisms of Brazilian Aedes aegypti populations from 2001 to 2004. Am J Trop Med Hyg. 2007;77:467-477.

9. da-Cunha MP, Lima JB, Brogdon WG, Moya GE, Valle D. Monitoring of resistance to the pyrethroid cypermethrin in Brazilian Aedes aegypti (Diptera: Culicidae) populations collected between 2001 and 2003. Mem Inst Oswaldo Cruz. 2005;100:441-444.

10. Shaalan EAS, Canyon D, Younes MWF, Abdel-Wahab H, Mansour AH. A review of botanical phytochemicals with mosquitocidal potential. Environ Int. 2005;31:1149-1166.

11. Schmutterer H. Properties and potential of natural pesticides from the neem tree, Azadirachta indica. Annu Rev Entomol. 1990;35: $197-271$.

12. Zebitz CPW. Effect of some crude and azadirachtin-enriched neem (Azadirachta indica) seed kernel extracts on larvae of Aedes aegypti. Entomol Exp Appl. 1984;35:1-16.

13. Gomes SA, Paula AR, Ribeiro A, et al. Neem oil increases the efficiency of the entomopathogenic fungus Metarhizium anisopliae for the control of Aedes aegypti (Diptera: Culicidae) larvae. Parasit Vectors. 2015;8:669. doi: 10.1186/s13071-015-1280-9.

14. Monzon RB, Alvior JP, Luczon LLC, Morales AS, Mutuc FES. Larvicidal potential of five Philippine plants against Aedes aegypti (Linneaeus) and Culex quinquefasciatus (Say). Southeast Asian J Trop Med Public Health. 1994;25:755-759.

15. Dua VK, Pandey AC, Raghavendra K, Gupta A, Sharma T, Dash AP. Larvicidal activity of neem oil (Azadirachta indica) formulation against mosquitoes. Malar J. 2009;8:124.

16. Mckenzie CL, Byford RL. Continuous, alternating, and mixed insecticides affect development of resistance in the horn fly (Diptera: Muscidae). J Econ Entomol. 1993;86:1040-1048.

17. Sparks RE. Microencapsulation. In: Kirk-Othmer. Encyclopedia of Chemical Technology. 3rd ed. New York: John Willey and Sons; v. 15; 1981: 470p. I.

18. Ré MI. Microencapsulation by spray drying. Drying Technol. 1998;16:1195-1236.

19. Park JK, Chang HN. Microencapsulation of microbial cells. Biotechnol Adv. 2000;18:303-319.

20. Risch SJ. Encapsulation: overview of uses and techniques. In: Risch SJ, Reineccius GA, editors. Encapsulation and Controlled Release of Food Ingredients. Washington, DC: ACS; 1995:2-7.

21. Amsden B. Solute diffusion within hydrogels. Mechanisms and models. Macromolecules. 1998;31(23):8382-8395.

22. Soliman E. Microencapsulation of essential oils within alginate: formulation and in vitro evaluation of antifungal activity. $J$ Encapsul Adsorp Sci. 2013;3(1):48-55.
23. Ceausoglu I, Hunkeler D. A new microencapsulation device for controlled membrane and capsule size distributions. J Microencapsul. 2002;19:725-735.

24. Liu CP, Liu SD. Formulation and characterization of the microencapsulated entomopathogenic fungus Metarhizium anisopliae MA126. J Microencapsul. 2009;26(5):377-384.

25. Sezer AD, Akbuga J. Release characteristics of chitosan treated alginate beads: II. Sustained release of a low molecular drug from chitosan treated alginate beads. J Microencapsul. 1999;16:687-696.

26. Pereira RM, Roberts DW. Alginate and cornstarch mycelial formulations of entomopathogenic fungi Beauveria bassiana and Metarhizium anisopliae. J Econ Entomol. 1991;84:1657-1661.

27. Paula HCB, Oliveira EF, Abreu FOMS, Paula RCM, Morais SM, Forte MMC. Esferas (beads) de alginato como agente encapsulante de óleo de Croton zehntneri Pax et Hoffm [Alginate beads for encapsulation of Croton zehntneri oil]. Polímeros. 2010;20:112-120. Portuguese.

28. WHO. Malaria: Vector control and insecticide resistance. Available from: http://www.who.int/malaria/areas/vector_control/en/. Accessed January 1, 2016.

29. Ranson H, Burhani J, Lumjuan N, Black WC. Insecticide resistance in dengue vectors. TropIKA net J. 2010;1:1.

30. Blanford S, Shi W, Christian R, et al. Lethal and pre-lethal effects of a fungal biopesticide contribute to substantial and rapid control of malaria vectors. PLoS One. 2011;6(8):e23591.

31. Howard AFV, N'Guessan R, Koenraadt CJM, et al. First report of the infection of insecticide-resistant malaria vector mosquitoes with an entomopathogenic fungus under field conditions. Malar J. 2011;10:24-32.

32. Farenhorst M, Mouatcho JC, Kikankie CK, et al. Fungal infection counters insecticide resistance in African malaria mosquitoes. Proc Natl Acad Sci USA. 2009;106(41):17443-17447.

33. Paula AR, Carolino AT, Paula CO, Samuels RI. The combination of the entomopathogenic fungus Metarhizium anisopliae with the insecticide imidacloprid increases virulence against the dengue vector Aedes aegypti (Diptera: Culicidae). Parasit Vectors. 2011;4:8-15.

34. Scholte EJ, Ng'habi K, Kihonda J, et al. An entomopathogenic fungus for control of adult African malaria mosquitoes. Science. 2005;308:1641-1642.

35. Farenhorst M, Farina D, Scholte EJ, et al. African water storage pots for the delivery of the entomopathogenic fungus Metarhizium anisopliae to the malaria vectors Anopheles gambiae s.s. and Anopheles funestus. Am J Trop Med Hyg. 2008;78(6):910-916.

36. Farenhorst M, Hilhorst A, Thoma MB, Knols BGJ. Development of fungal applications on netting substrates for malaria vector control. J Med Entomol. 2011;48(2):305-313.

37. Hecht $\mathrm{O}$, Hernandez-Corzo J. On the visual orientation of mosquitoes in their search of resting places. Entomol Exp Appl. 1963;6:63-74.

38. Carolino AT, Paula AR, Silva CP, Butt TM, Samuels RI. Monitoring persistence of the entomopathogenic fungus Metarhizium anisopliae under simulated field conditions with the aim of controlling adult Aedes aegypti (Diptera: Culicidae). Parasit Vectors. 2014;7:198-204.

39. Paula AR, Brito ES, Pereira CR, Carrera MP, Samuels RI. Susceptibility of adult Aedes aegypti (Diptera: Culicidae) to infection by Metarhizium anisopliae and Beauveria bassiana: prospects for Dengue vector control. Biocont Sci Tech. 2008;18:1017-1025.

40. Paula AR, Carolino AT, Silva CP, Pereira CR, Samuels RI. Testing fungus impregnated cloths for the control of adult Aedes aegypti under natural conditions. Parasit Vectors. 2013;6:256-261.

41. Mnyone LL, Kirby MJ, Lwetoijera DW, et al. Tools for delivering entomopathogenic fungi to malaria mosquitoes: effects of delivery surfaces on fungal efficacy and persistence. Malar J. 2010;9:246-252.

42. Mnyone LL, Lyimo IN, Lwetoijera DW, et al. Exploiting the behaviour of wild malaria vectors to achieve high infection with fungal biocontrol agents. Malar J. 2012;11:87-97.

43. Lwetoijera DW, Sumaye RD, Madumla EP, et al. An extra-domiciliary method of delivering entomopathogenic fungus, Metarhizium anisopliae IP 46 for controlling adult populations of the malaria vector, Anopheles arabiensis. Parasit Vectors. 2010;3:18-23. 
44. Butt TM, Jackson C, Magan N, editors. Fungi as Biocontrol Agents: Progress Problems and Potential. UK: CABI Publishing; 2001:1-8.

45. Darbro JM, Johnson PH, Thomas MB, Ritchie SA, Kay BH, Ryan PA Effects of Beauveria bassiana on survival, blood-feeding success, and fecundity of Aedes aegypti in laboratory and semi-field conditions. Am J Trop Med Hyg. 2012;86:656-664.

46. Santos A, Oliveira BL, Samuels RI. Selection of entomopathogenic fungi for use in combination with sub-lethal doses of imidacloprid. Mycopathologia. 2007;163:233-240.

47. Sim S, Dimopoulos G. Dengue virus inhibits immune responses in Aedes aegypti cells. PLoS One. 2010;5:e10678.

48. Boucias DG, Stokes C, Storey G, Pendland JC. The effects of imidacloprid on the termite Reticulitermes flavipes and its interaction with the mycopathogen Beauveria bassiana. Pflanzenschutz-Nachrichten Bayer. 1996;49:103-144.
49. Quintela ED, McCoy CW. Conidial attachment of Metarhizium anisopliae and Beauveria bassiana to the larval cuticle of Diaprepes abbreviatus (Coleoptera: Curculionidae) treated with imidacloprid. J Invertebr Pathol. 1998;72:220-230.

50. Pridgeon JW, Pereira RM, Becnel JJ, Allan SA, Clark GG, Linthicum KJ. Susceptibility of Aedes aegypti, Culex quinquefasciatus say, and Anopheles quadrimaculatus say to 19 pesticides with different modes of action. J Med Entomol. 2008;45:82-87.

51. Paul A, Harrington LC, Scott JG. Evaluation of novel insecticides for control of dengue vector Aedes aegypti (Diptera: Culicidae). J Med Entomol. 2006;43:55-60.

\section{Publish your work in this journal}

Open Access Insect Physiology is an international, peer-reviewed, open access journal publishing original research, reports, reviews and commentaries on all areas of insect physiology. The manuscript management system is completely online and includes a very quick and fair peer-review system, which is all easy to use.

\section{Dovepress}

Visit http://www.dovepress.com/testimonials.php to read real quotes from published authors. 